\title{
Dissociation between fatty liver and insulin resistance: the role of adipose triacylglycerol lipase
}

\author{
N. Stefan $\cdot$ H. Staiger $\cdot$ H.-U. Häring
}

Received: 9 September 2010 /Accepted: 13 September 2010 /Published online: 16 October 2010

(C) Springer-Verlag 2010

\begin{abstract}
Non-alcoholic fatty liver disease (NAFLD) is strongly associated with insulin resistance and type 2 diabetes in humans. Ongoing research aims to clarify the mechanisms involved in this relationship. Studying pathways that are involved in the dissociation between fatty liver and insulin resistance may help to achieve this goal. Among several enzymes that regulate the fate of hepatic lipids, adipose triacylglycerol lipase (ATGL) is of interest. This article briefly summarises novel information about the impact of ATGL in this process.
\end{abstract}

Keywords Hepatic de novo lipogenesis · Insulin resistance $\cdot$ Non-alcoholic fatty liver disease $\cdot$ Prediabetes . Triacylglycerol hydrolysis · Triacylglycerol synthesis . Type 2 diabetes

$\begin{array}{ll}\text { Abbreviations } \\ \text { ATGL } & \text { Adipose triacylglycerol lipase } \\ \text { CGI-58 } & \text { Comparative gene identification-58 } \\ \text { DAG } & \text { Diacylglycerol } \\ \text { NAFLD } & \text { Non-alcoholic fatty liver disease } \\ \text { PKC } & \text { Protein kinase C } \\ \text { TAG } & \text { Triacylglycerol }\end{array}$

N. Stefan $(\bowtie) \cdot$ H. Staiger $\cdot$ H.-U. Häring

Department of Internal Medicine,

Division of Endocrinology, Diabetology,

Vascular Medicine,

Nephrology and Clinical Chemistry,

Member of the Deutsches Zentrum für Diabetesforschung (DZD),

University of Tübingen,

Otfried-Müller-Str. 10,

D-72076 Tübingen, Germany

e-mail: norbert.stefan@med.uni-tuebingen.de

\section{Causes and metabolic consequences of non-alcoholic fatty liver disease}

Non-alcoholic fatty liver disease (NAFLD) is strongly associated with type 2 diabetes in humans. Both conditions are accompanied by behavioural factors that arise from our modern lifestyle. In particular, an increased energy intake is thought to represent a major factor in their pathogenesis. In addition, a diet containing large amounts of saturated and trans-unsaturated fatty acids, and the increase in consumption of high-fructose corn syrup in the westernised world, have contributed to the respective increases in the incidences of NAFLD and type 2 diabetes. Furthermore, a sedentary lifestyle with reduced physical activity, independent of diet, represents another determinant for both diseases [1-3]. From a mechanistic point of view, the relationship between NAFLD and type 2 diabetes is thought to result predominantly from hyperinsulinaemia, which is usually present before and at the time of the diagnosis of diabetes. Skeletal muscle insulin resistance-mediated hyperglycaemia is thought to result in insulin hypersecretion from the beta cells; hyperinsulinaemia then induces hepatic lipogenesis via activation of sterol-regulatory binding protein $1 \mathrm{c}$ (SREBP-1c), a transcription factor regulating the expression of enzymes involved in the synthesis of fatty acids in the liver. Furthermore, hyperglycaemia in prediabetes and diabetes adds to this process via activation of carbohydrate response element-binding protein, which exerts, similarly to SREBP-1c, stimulatory effects on the expression of genes involved in lipogenesis and triacylglycerol (TAG) synthesis. In addition, expansion of adipose tissue and a disproportionate fat distribution, particularly with increased visceral adiposity that is strongly associated with insulin resistance, releasing humoral factors that regulate liver fat (e.g. adiponectin), are relevant. Finally, impaired hepatic 
lipid oxidation as well as dysregulated lipogenesis, factors that are affected by genetics, may be of pathophysiological relevance $[2,3]$. Consequently, fatty liver may rather be a consequence or marker of insulin resistance rather than a cause. However, mechanistic proof for the role of fatty liver in the pathogenesis of increased hepatic glucose production has come from animal and in vitro studies. These studies have provided evidence that in hepatic steatosis there is an accumulation of NEFA and TAG metabolites (fatty acyl CoA, diacylglycerol [DAG], ceramides). Thus, DAG may activate protein kinase $\mathrm{C}$ (PKC) isoforms, which have been shown to phosphorylate serine residues in insulin receptor substrate (IRS)-1/2. Serine phosphorylation of IRS is believed to inhibit insulin signal transduction $[4,5]$.

Finding such mechanistic proof in humans is more difficult. Liver fat measured directly in liver biopsies or indirectly by ${ }^{1} \mathrm{H}$-magnetic resonance spectroscopy is closely and positively correlated with measures of total and visceral adiposity, such as body mass index and waist circumference, making it difficult to distinguish between the possible impacts of liver fat vs adipose tissue on metabolism. However, in recent years fatty liver has been shown to correlate with insulin resistance, independent of other risk factors such as total and visceral adiposity [6-8]. Moreover, liver fat correlates more strongly with insulin resistance [6,7] and prediabetes [9] than with total and visceral fat mass, providing support for the notion that fatty liver is a condition that may have a dominant role in the pathogenesis of insulin resistance. Evidence from human studies on the metabolic consequences of the liver-secreted proteins fetuin-A and sex hormone-binding globulin, which are causally involved in the pathogenesis of insulin resistance and type 2 diabetes [10-13], support this hypothesis. Thus, studying the causes and metabolic consequences of fatty liver has become a major field of research for scientists investigating the pathogenesis of type 2 diabetes.

\section{Adipose TAG lipase and NAFLD}

In this issue of Diabetologia Turpin et al. [14] hypothesised in a study that targeting adipose TAG lipase (ATGL; also known as desnutrin, calcium-independent phospholipase A2 and patatin-like phospholipase domain-containing 2) in rodents would lead to changes in hepatic steatosis and concomitantly, insulin resistance. Their assumption was based on the fact that TAG accumulates in the hepatocytes, not only because of impaired hepatic lipid oxidation and/or increased lipid uptake, but also because of defective mobilisation of hepatic TAG. Among the lipases that are involved in the mobilisation of TAG (ATGL, hormone sensitive lipase and monoacylglycerol lipase), ATGL selec- tively performs the first step in TAG hydrolysis. ATGL cleaves TAG, but not DAG or monoacylglycerols, and is therefore considered to initiate the hydrolysis of stored lipids [15] (Fig. 1).

Consequently and as expected, ATGL-deficient mice accumulate large amounts of lipids in several tissues, including the liver [16]. In agreement with this finding, adenoviral overexpression of Atgl (also known as Pnpla2) reduced liver TAG in mice [17]. Thus, one may hypothesise that insulin sensitivity deteriorates in parallel to the increase in TAG content in ATGL deficiency. However, somewhat unexpectedly, increased whole body insulin sensitivity was found in Atgl-null mice [16]. To study the impact of ATGL deficiency in the liver on liver fat and insulin sensitivity, Turpin et al. isolated hepatocytes from ATGL-deficient and wild type mice. They found increased TAG content and fatty acid uptake, and decreased fatty acid oxidation, in the hepatocytes from ATGL-deficient mice. However, this was not accompanied by altered insulin sensitivity in these hepatocytes. In agreement with this, when the authors increased ATGL specifically in the liver of obese mice they found a large decrease in liver steatosis; however, they observed merely a mild increase in insulin sensitivity.

Very similar results were published recently by Brown et al. [18] using antisense oligonucleotides to inhibit expression of the comparative gene identification-58 (Cgi-58 [also known as $A b h d 5])$. The protein CGI-58, also known as $\alpha / \beta$

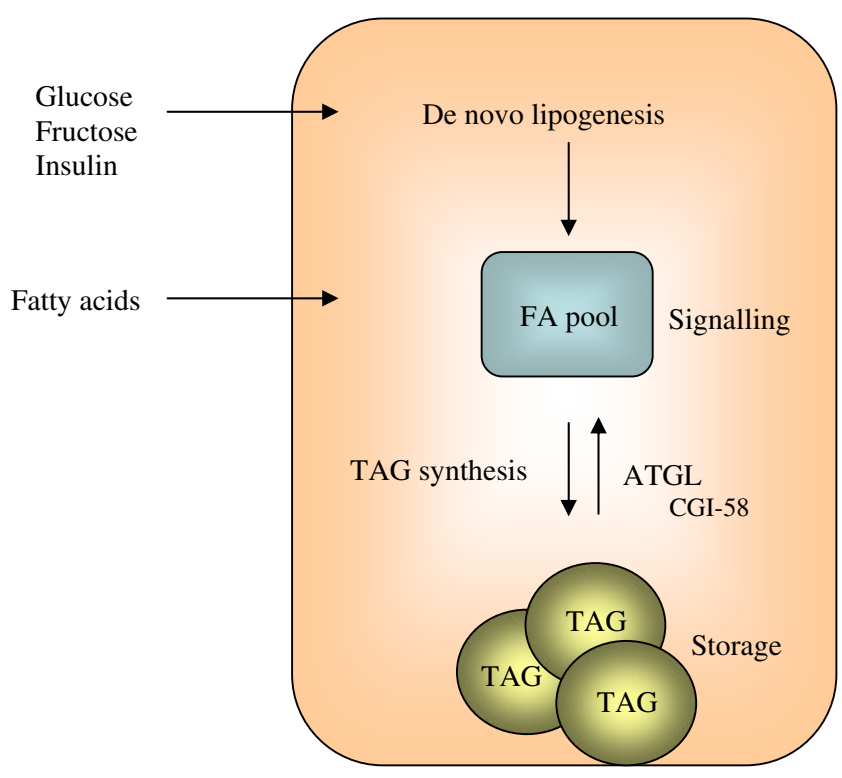

Fig. 1 A simplified hypothetical depiction of the role of ATGL in hepatic steatosis. A hyperenergetic diet, particularly one with an abundance of glucose and fructose, results in increased hepatic de novo lipogenesis, which together with high levels of circulating fatty acids (FA), increases the hepatic FA pool. Enzymes involved in TAG synthesis (e.g. DAG acyltransferases) induce hepatic TAG synthesis, thereby, decreasing FA signalling. ATGL and its co-activator CGI-58 are key regulators of TAG hydrolysis 
hydrolase domain-containing protein 5 , modulates TAG hydrolysis by acting as a co-activator for ATGL. The authors found that knockdown of $C g i-58$ in mice resulted in a large increase in hepatic TAG content, yet in a decrease in glucose intolerance and insulin resistance. Interestingly, this was accompanied by an increase in hepatic DAG and ceramides. Considering in particular the role of DAG in the induction of hepatic insulin resistance, again this finding was not expected. It cannot be ruled out that knockdown of Cgi-58 may have resulted in metabolic alterations in nonadipose tissue that may have caused the observed phenotype. In addition, as discussed by Zechner et al. [15], the stereospecificity of ATGL for the chemically distinct ester bonds within the TAG molecule is not known. Therefore, it is unclear whether the DAG generated by ATGL is involved in $s n-1,2-\mathrm{DAG}$ signalling, which is required for the activation of $\mathrm{PKC}$ isoenzymes.

\section{Conclusion}

The mechanism responsible for the observed dissociation between fatty liver and insulin resistance when targeting ATGL is not fully understood. It is possible that an ATGLmediated increase in TAG synthesis prevents fatty acidinduced lipotoxicity. Studying the regulation of the production and activation of ATGL may help us gain a better understanding of the mechanisms involved in hepatic lipid formation and breakdown, as well of hepatic lipid signalling and ultimately the pathogenesis of NAFLD.

Acknowledgements N. Stefan is currently supported by a Heisenberg grant from the Deutsche Forschungsgemeinschaft (STE 1096/1-1).

Duality of interest The authors declare that there is no duality of interest associated with this manuscript.

\section{References}

1. Angulo P (2002) Nonalcoholic fatty liver disease. N Engl J Med 346:1221-1231

2. Stefan N, Kantartzis K, Haring HU (2008) Causes and metabolic consequences of fatty liver. Endocr Rev 29:939-960
3. Choi SS, Diehl AM (2008) Hepatic triglyceride synthesis and nonalcoholic fatty liver disease. Curr Opin Lipidol 19:295300

4. Samuel VT, Petersen KF, Shulman GI (2010) Lipid-induced insulin resistance: unravelling the mechanism. Lancet 375:22672277

5. Fritsche L, Weigert C, Häring HU, Lehmann R (2008) How insulin receptor substrate proteins regulate the metabolic capacity of the liver-implications for health and disease. Curr Med Chem $15: 1316-1329$

6. Stefan N, Kantartzis K, Machann J et al (2008) Identification and characterization of metabolically benign obesity in humans. Arch Intern Med 168:1609-1616

7. Fabbrini E, Magkos F, Mohammed BS et al (2009) Intrahepatic fat, not visceral fat, is linked with metabolic complications of obesity. Proc Natl Acad Sci USA 106:15430-15435

8. Tiikkainen M, Bergholm R, Vehkavaara S et al (2003) Effects of identical weight loss on body composition and features of insulin resistance in obese women with high and low liver fat content. Diabetes 52:701-707

9. Kantartzis K, Machann J, Schick F, Fritsche A, Häring HU, Stefan N (2010) The impact of liver fat vs visceral fat in determining categories of prediabetes. Diabetologia 53:882889

10. Stefan N, Fritsche A, Weikert C et al (2008) Plasma fetuin-A levels and the risk of type 2 diabetes. Diabetes 57:2762-2767

11. Ding EL, Song Y, Manson et al (2009) Sex hormone-binding globulin and risk of type 2 diabetes in women and men. N Engl J Med 361:1152-1163

12. Stefan N, Schick F, Haring HU (2009) Sex hormone-binding globulin and risk of type 2 diabetes. N Engl J Med 361:26752676

13. Peter A, Kantartzis K, Machann J et al (2010) Relationships of circulating sex hormone-binding globulin with metabolic traits in humans. Diabetes. doi:10.2337/db10-0179

14. Turpin SM, Hoy AJ, Brown R et al (2010) Adipose triglyceride lipase is a major regulator of hepatic lipid metabolism but not insulin sensitivity in mice. Diabetologia. doi:10.1007/s00125-0101895-5

15. Zechner R, Kienesberger PC, Haemmerle G, Zimmermann R, Lass A (2009) Adipose triglyceride lipase and the lipolytic catabolism of cellular fat stores. J Lipid Res 50:3-21

16. Haemmerle G, Lass A, Zimmermann R et al (2006) Defective lipolysis and altered energy metabolism in mice lacking adipose triglyceride lipase. Science 312:734-737

17. Reid BN, Ables GP, Otlivanchik OA et al (2008) Hepatic overexpression of hormone-sensitive lipase and adipose triglyceride lipase promotes fatty acid oxidation, stimulates direct release of free fatty acids, and ameliorates steatosis. J Biol Chem 283: 13087-13099

18. Brown JM, Betters JL, Lord C et al (2010) CGI-58 knockdown in mice causes hepatic steatosis, but prevents diet-induced obesity and glucose intolerance. J Lipid Res. doi:10.1194/j1r.M010256 